\title{
AUTOMATIC IDENTIFICATION OF VIETNAMESE DIALECTS
}

\author{
PHAM NGOC HUNG ${ }^{1,2}$, TRINH VAN LOAN ${ }^{1,2}$, NGUYEN HONG QUANG ${ }^{2}$ \\ ${ }^{1}$ Faculty of Information Technology, Hung Yen University of Technology and Education, \\ ${ }^{2}$ School of Information and Communication Technology, Hanoi University of Science and \\ Technology \\ 1,2pnhung@utehy.edu.vn; 1,2 loantv@soict.hust.edu.vn; ${ }^{2}$ quangnh@soict.hust.edu.vn
}

\begin{abstract}
The dialect identification has been under study for many languages over the world nevertheless the research on signal processing for Vietnamese dialects is still limited and there are not many published works. There are many different dialects for Vietnamese. The influence of dialectal features on speech recognition systems is important. If the information about dialects is known during speech recognition process, the performance of recognition systems will be better because the corpus of these systems is normally organized according to different dialects. In our experiments, MFCC coefficients, formants, correspondent bandwidths and the fundamental frequency with its variants are input parameters for GMM. The experiment result for the dialect corpus of Vietnamese shows that the performance of dialectal identification with baseline increases from $58.6 \%$ for the case using only MFCC coefficients to $70.8 \%$ for the case using MFCC coefficients and the information of fundamental frequency. By combining the formants and their bandwidths with the normalized $F 0$ according to average and standard deviation $F 0$, the best recognition rate is $72.2 \%$.
\end{abstract}

Keywords. Fundamental frequency, MFCC, Formant, Bandwidth, GMM, Vietnamese dialects, identification.

\section{INTRODUCTION}

Vietnamese is a tonal language with many different dialects. It is the diversity of Vietnamese dialects that remains a great challenge to the systems of Vietnamese recognition. In other words, the pronunciation modality of the word is not the same from locality to locality. For example, for two Vietnamese dialects, the sound may be heard as the same but the sense is quite different depending on the dialect. This can reduce the performance of recognition systems if these systems have no information and training data of each dialect to be recognized.

For many languages in the world such as English [1], Chinese [2], Thai [3], Hindi [4] .. there are already studies on the dialect identification. For Vietnamese, the studies on dialects have been carried out for a long time ago but mainly on the linguistic approach and were still limited on the signal processing approach. Therefore, the research and the solution for Vietnamese dialect identification are quite necessary to improve the performance of Vietnamese recognition systems.

This paper presents the research results on Vietnamese dialectal identification based on GMM (Gaussian Mixture Model) using MFCC (Mel-frequency Cepstral Coefficients) and 
tonal features through the variation of fundamental frequency. The identification experiments were performed with the corpus VDSPEC (Vietnamese Dialect Speech Corpus) built for the research on Vietnamese dialects. VDSPEC consists of 150 speakers with the duration of 45.12 hours. Section 2 of the paper describes the overview of Vietnamese dialects. GMM model, MFCC, formants and fundamental frequency F0 used in this model are presented in section 3. The experiments and identification results are given in section 4. Finally, section 5 is conclusions and development orientations.

\section{AN OVERVIEW OF THE VIETNAMESE DIALECTS}

It is known that a dialect is a form of the language spoken in different regions of the country. These dialects may have distinctions of words, grammar, and pronunciation modalities. Vietnamese is the language that has many dialects.

The division of the Vietnamese dialects has been done by Vietnamese linguists with some different opinions. Nevertheless, the majority of linguists think that Vietnamese can be divided into three main dialects: northern dialect corresponding to Tonkin, central dialect corresponding to areas from Thanh Hoa province to Hai Van pass, southern dialect corresponding to areas from Hai Van pass to southern provinces [5]. In any case, this division is only relative because the geographical boundaries to divide the dialects are not completely clear. In fact, for the same regions, dialect can vary from a village to another. For three principal dialects above, in addition to the significant differences in vocabulary, it makes the listener easily perceive, distinguish between the dialects that is pronunciation modality. Phonetics of three main dialects differs significantly. For Vietnamese tone system, northern dialect has full six tones including level tone ("thanh ngang"), low-falling tone ("thanh huyền"), asking tone ("thanh hói"), rising tone ("thanh sắc"), broken tone ("thanh ngã") and heavy tone ("thanh năng"), while central dialect has only five tones. For Thanh Hoa, Quang Binh, Quang Tri, Thua Thien voices and southern voice in general, there is no distinction between asking tone and broken tone. For Nghe An and Ha Tinh voices, broken tone and heavy tone are the same. In terms of prosody, three main dialects are entirely different.

The number of different Vietnamese dialects is very big. Traditionally, Vietnam is divided geographically into three regions: North, Centre and South. The dialects for these three regions are also different both local vocabularies and pronunciation modalities. That is why we have chosen three representative dialects for these regions.

In our research, it is the difference between pronunciation modalities and but not local vocabulary that is exploited to identify three main dialects.

\section{GMM WITH MFCC, FORMANTS AND F0}

Multivariate Gaussian Mixture Model has been used for speaker recognition [6], English dialect recognition [7], Chinese dialect recognition [8] and language identification [9, 10]. Supper vectors [11] are also used in research on dialect identification with positive results. To explain why GMM often used in speaker recognition, language, and dialect identification... one can deduce as follows. Even in the cases where content cannot be understood clearly, people still have the ability to sense voice, language or dialect that they have known already. 
In that case, general information or information envelope on phonetics has helped people recognize the voice, language and dialect without the need for more information in detail about the content that the speaker transmits. By taking a large enough number of Gaussian distribution components, adjusted average and variance of them as well as weights in a linear combination, GMM can approximate most continuous distribution density with optional precision. Therefore, GMM allows modeling only the basic distribution of the speaker's phonetic or the perception of phonetic information envelope mentioned above. The average while determining GMM model can eliminate the factors that affect the acoustic features like phonetic variation over time of different speakers and retain only what is an essential characteristic of the voice of a region such as in the case of dialect identification.

A multivariate Gaussian mixture model is a weighted sum of $M$ Gaussian density components as the following formulas:

$$
p(X \mid \lambda)=\sum_{i=1}^{M} \pi_{i} g_{i}\left(X \mid \mu_{i}, \Sigma_{i}\right),
$$

where $\boldsymbol{X}$ is a data vector containing the parameters of object which needs to be represented, $\pi_{i}, i=1, \ldots, M$, is mixture weight, $g_{i}\left(X \mid \mu_{i}, \Sigma_{i}\right)$ is a component Gaussian density function as the formulas (2) with average vector $\mu_{i}$ of $D$ dimension vector and $D \times D$ dimension covariance matrix $\Sigma_{i}$ :

$$
g_{i}\left(X \mid \mu_{i}, \Sigma_{i}\right)=\frac{1}{(2 \pi)^{\frac{D}{2}}\left|\Sigma_{i}\right|^{\frac{1}{2}}} \exp \left\{-\frac{1}{2}\left(X-\mu_{i}\right)^{\prime} \Sigma_{i}^{-1}\left(X-\mu_{i}\right)\right\} .
$$

The mixture weights must meet the condition $\Sigma_{i=1}^{M} \pi_{i}=1$.

A full GMM is parameterized by average vectors, covariance matrix and mixture weights of all Gaussian components. These parameters can be represented in a shortened form (3)

$$
\lambda=\left\{\pi_{i}, \mu_{i}, \Sigma_{i}\right\}, i=1,2, \ldots, M .
$$

To identify dialects, each dialect is represented by a GMM and is referenced to the model $\lambda$ of this model. In the case where MFCC are used as feature vectors, the spectral envelope of the $i^{t h}$ acoustic class is represented by average $\mu_{i}$ of the $i^{t h}$ component and the variation of spectral envelope is represented by covariance matrix $\Sigma_{i}$.

Assuming $T$ is the number of feature vectors ( $T$ is also the number of speech frames), $M$ is the number of Gaussian components

$$
X=\left\{x_{1}, x_{2}, \ldots, x_{T}\right\} .
$$

GMM likelihood is

$$
p(X \mid \lambda)=\prod_{t=1}^{T} p\left(x_{t} \mid \lambda\right) .
$$

The expression (5) is a nonlinear function for $\lambda$ so it cannot be maximized directly and the maximized likelihood parameters can be received using the EM (expectation - maximization) algorithm. 
The idea of this algorithm is that beginning with the initial model $\lambda$, to estimate a new model $\bar{\lambda}$ so that:

$$
p(X \mid \bar{\lambda}) \geq p(X \mid \lambda) .
$$

This new model is an initial model for the next iteration and the process is repeated until the convergence threshold is achieved. In fact, expectation maximization algorithm attempts to find the $\lambda$ that maximizes the $\log$ probability $\log p(X \mid \lambda)$ of the data $\boldsymbol{X}$.

In a study published in [12], GMM is used only with the parameters MFCC. The computation of theses parameters is described in Figure 1.

In Figure 1, speech signal is framed with frame length $0.01 \mathrm{~s}$ and frame shift $0.005 \mathrm{~s}$. The emphasis filter has input-output relationship:

$$
y(n)=x(n)-0.96 x(n-1) .
$$

Then speech signal is passed through Hamming window with window length $N$

$$
w(n)=0.54-0.46 \cos (2 \pi n /(N-1)) \text { with } 0 \leq n \leq N-1 .
$$

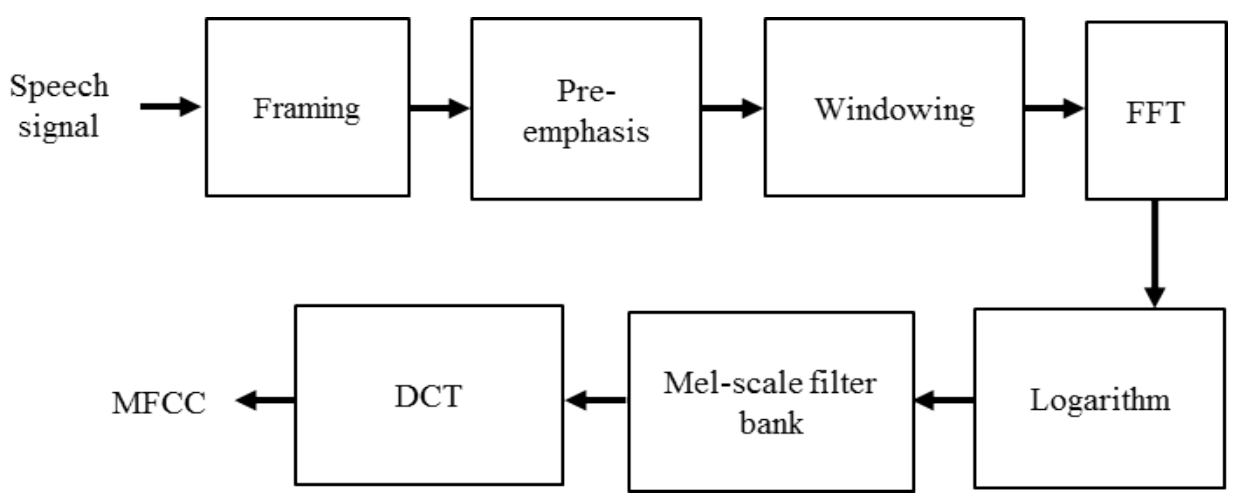

Figure 1. Computation of MFCC parameters

FFT (Fast Fourier Transform) is applied for windowed signal and the signal spectrum goes through Mel scale triangle filter bank. MFCC are received after DCT (Discrete Cosine Transform).

Besides MFCC, the formants and corresponding bandwidths are also used as input features for GMM.

Next, the paper presents dialect identification method based on GMM with the combination of MFCC, formants, $F 0$ and its variants. The experiments are carried out using open source tools ALIZE [6]. The $F 0$ values of each frame are added at the end of feature vectors.

\section{EXPERIMENT RESULTS}

\subsection{Speech data for experiment}

The speech corpus VDSPEC is used for the experiments. Speech is recorded by reading the text organized according to 6 topics with tonal balance (The number of words is equal for each tone and equals 717 on average). 
The sampling frequency is $16000 \mathrm{~Hz}$ and 16 bits per sample. The speaker's average age is 21. At this age, voice quality is steady with full features for the local voice. Each dialect has 50 speakers including 25 men and 25 women. Hanoi voice is chosen for northern dialect, Hue voice for central dialect and Ho Chi Minh City voice for southern dialect. For each topic, the speaker reads 25 sentences and a sentence's length is about 10 seconds. The recording duration is 45.12 hours with the volume $4.84 \mathrm{~GB}$. Some information of VDSPEC is given in the Tables 1 and 2 .

Table 1. Statistics according to the dialects of VDSPEC

\begin{tabular}{|l|l|l|}
\hline Dialect & No. Sentences & Duration (h) \\
\hline Northern & 6250 & 14.41 \\
\hline Central & 6250 & 14.65 \\
\hline Southern & 6250 & 16.06 \\
\hline Total & $\mathbf{1 8 7 5 0}$ & $\mathbf{4 5 . 1 2}$ \\
\hline
\end{tabular}

Table 2. Statistics according to the topics of VDSPEC

\begin{tabular}{|l|l|l|l|}
\hline Topic & No. Sentences & No. Syllables & Duration (h) \\
\hline Life & 3750 & 855 & 9.96 \\
\hline Science & 3750 & 893 & 8.03 \\
\hline Business & 3750 & 729 & 10.06 \\
\hline Car-motorbike & 3750 & 652 & 7.31 \\
\hline Law & 3750 & 855 & 9.76 \\
\hline Total & $\mathbf{1 8 7 5 0}$ & $\mathbf{3 9 8 4}$ & $\mathbf{4 5 . 1 2}$ \\
\hline
\end{tabular}

For experiments, the above corpus is divided into five parts. With each dialect, 10 speakers ( 5 male voices and 5 female voices) were used for testing, 40 speakers (20 male voices and 20 female voices) for training. All of the test experiments are performed using cross-validation.

\subsection{Selection of the number of coefficients MFCC}

To find out the best number of coefficients MFCC used for dialect identification regardless of gender, the number of coefficients MFCC is varied from 5 to 19. The experiments are carried out for each dialect then the average value is taken. The Gaussian component number $M$ is 20 for this experiment and the following experiments take this value as the baseline. The next experiment in 4.5 will take the different values of the Gaussian component number for the examination of its impact on performance All of the tests in our experiments are speaker independent.

Figure 2 shows that the maximal average value for the number of coefficients MFCC is 10 with score 8. In this case, the score is the highest likelihood for each dialect. However, with this value there is a great disparity in the scores of dialect identification for three dialects. Two additional values of the number of coefficients MFCC can be selected. The first value is 13. Three curves intersect in this value. The scores at these values are not highest but 
are equal. The second value is 11 . For this value, the average score is higher in comparison with the value 13 except for central dialect and the score of this dialect is a little bit lower. Finally, two values 11 and 13 are chosen for the next experiments.

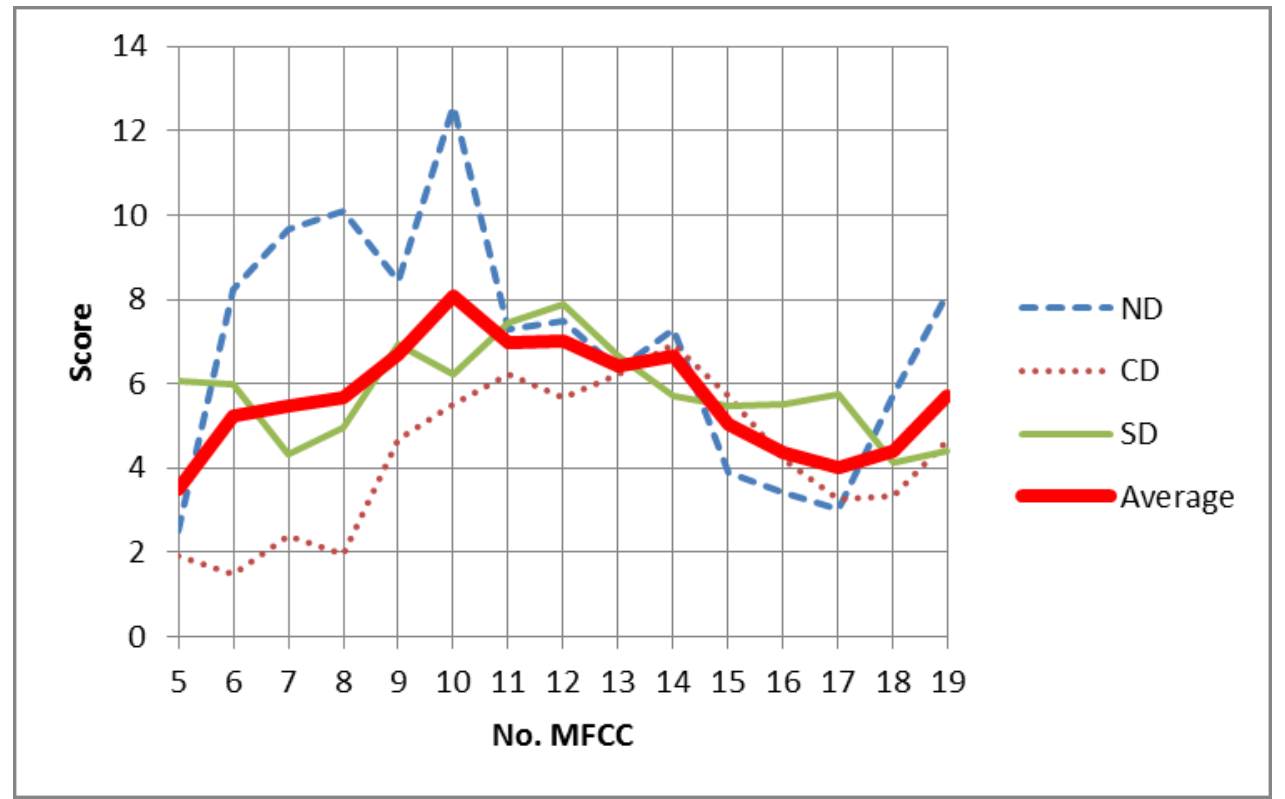

Figure 2. Experiments for selecting the number of MFCC. ND: Northern Dialect, CD: Central Dialect, SD: Southern Dialect

\subsection{Combination of MFCC coefficients and F0 parameters}

In [13], the different variation of $F 0$ for three dialects have been evaluated. Generally speaking, the direction and the range of $F 0$ variation for Hue tones tend to be opposed to Hanoi tones. Unless broken tone, the trend of $F 0$ variations for Ho Chi Minh City voices is rather close to Hanoi voices. The $F 0$ variations of broken tone for Ho Chi Minh City voices tend to go up like the asking tone of Hanoi voices. These distinctions can be used as the important features for identifying the dialects.

For this case, MFCC coefficients are combined with fundamental frequency $F 0, \log F 0(t)$ and normalized values of $F 0$ and $\log F 0(t)$. Beside $F 0$ value, some quantities derived from $F 0$ are calculated as follows

The derivative $F 0($ diff $F 0(t))$ :

$$
\operatorname{diffF0}(t)=d F 0(t) / d t .
$$

The trend upward or downward of $F 0$ for each sentence $(c d F 0(t))$ :

$$
c d F 0(t)=\left\{\begin{array}{cl}
-1 & \text { if }\left(\left(F 0_{i}-F 0_{i-1}\right) \leq-3\right) \\
0 & \text { if }\left(-3<\left(F 0_{i}-F 0_{i-1}\right)<3\right) \\
1 & \text { if }\left(\left(F 0_{i}-F 0_{i-1}\right) \geq 3\right)
\end{array}\right.
$$


The normalized $F 0$ according to average $F 0$ for each sentence $(F 0 s b M(t))$ :

$$
F 0 \operatorname{sbM}(t)=F_{0}(t) / \overline{F_{0}(t)}
$$

The normalized $F 0$ according to average and standard deviation $F 0(F 0 s b M S D(t))$ :

$$
F 0 \operatorname{sbMSD}(t)=\frac{F_{0}(t)-\overline{F_{0}(t)}}{\sigma F_{0}(t)} .
$$

The derivative $\log F 0(t)(\operatorname{diff} \log F 0(t))$ :

$$
\operatorname{diff} \log F 0(t)=d \log F 0(t) / d t .
$$

The normalized $\log F 0(t)$ according to $\min \log F 0(t)$ and $\max \log F 0(t)$ for each sentence $(\log F 0 \operatorname{sbMM}(t))$ :

$$
\log F 0 \operatorname{sbMM}(t)=\frac{\log F_{0}(t)-\min \log F_{0}(t)}{\max \log F_{0}(t)-\min \log F_{0}(t)}
$$

The normalized $\log F 0(t)$ according to average $\log F 0(t)$ for each sentence $(\log F 0 \operatorname{sb} M(t))$ :

$$
\log F 0 \operatorname{sbM}(t)=\log F_{0}(t) / \overline{\log F_{0}(t)} .
$$

\begin{tabular}{|c|c|c|c|}
\hline Index & $\begin{array}{l}\text { MFCC } \\
+F 0 \text { Parameters }\end{array}$ & $\begin{array}{l}\text { Recognition } \\
\text { Rate } \\
(\mathrm{MFCC}=11)\end{array}$ & $\begin{array}{l}\text { Recognition } \\
\text { Rate } \\
(\mathrm{MFCC}=13)\end{array}$ \\
\hline 1 & MFCC & $59.6 \%$ & $58.6 \%$ \\
\hline 2 & $F 0$ & $65.1 \%$ & $64.4 \%$ \\
\hline 3 & $\operatorname{diffF0}(t)$ & $62.9 \%$ & $61.9 \%$ \\
\hline 4 & $c d F 0(t)$ & $60.6 \%$ & $58.1 \%$ \\
\hline 5 & $F 0 \operatorname{sbMM}(t)$ & $61.4 \%$ & $60.9 \%$ \\
\hline 6 & $F 0 \operatorname{sbM}(t)$ & $69.5 \%$ & $70.8 \%$ \\
\hline 7 & $F 0 s b M S D(t)$ & $62.9 \%$ & $61.9 \%$ \\
\hline 8 & $\operatorname{LogF0}(t)$ & $68.6 \%$ & $68.4 \%$ \\
\hline 9 & $\operatorname{diff} \log F 0(t)$ & $59.5 \%$ & $57.3 \%$ \\
\hline 10 & $\operatorname{LogF0sbMM(t)}$ & $61.0 \%$ & $60.2 \%$ \\
\hline 11 & $\operatorname{LogF} 0 \operatorname{sbM}(t)$ & $69.7 \%$ & $68.8 \%$ \\
\hline 12 & $\operatorname{LogF0sbMSD(t)}$ & $59.5 \%$ & $57.3 \%$ \\
\hline
\end{tabular}

The normalized $\log F 0$ according to average and standard deviation $\log F 0(t)(\log F 0 M S D(t))$ :

$$
\log F 0 M S D(t)=\frac{\log F_{0}(t)-\overline{\log F_{0}(t)}}{\sigma \log F_{0}(t)} .
$$

Table 3. Recognition results using $\mathrm{MFCC}=11, \mathrm{MFCC}=13$ and $F 0$ parameters 
Praat ${ }^{1}$ was used to estimate fundamental frequency variations for Vietnamese tones in VDSPEC. In Table 3, the second column (Parameters) shows the parameters that were used in the model. For the first row, the only MFCC coefficients are used. With 11 coefficients of MFCC, the highest recognition rate is $69.5 \%$ for the case (6) and $69.7 \%$ for the case (11). This shows that the recognition performance is better if the F0 information is added (The score increases approximately 10\%).

In the last column of Table 3 where $\mathrm{MFCC}=13$, without $F 0$ parameters, the recognition rate is only $58.6 \%$. The highest recognition rate is $70.8 \%$ for the case where MFCC are combined with $F 0 s b M$. This is also consistent with the case MFCC $=11$. With the combination of MFCC and $F 0$, the recognition rates are improved significantly (up $12.2 \%$ ) in comparison with the case without $F 0$ information.

The confusion matrix without gender distinction with the combination of MFCC and F0 is given in Table 4. In general, Table 4 shows that central dialect tends to reach more northern dialect, and southern dialect tends to reach more central dialect. This is consistent with the fact that northern and central dialects have many similarities and the pronunciation modality is almost the same in most of the tones. The more geographical distance is far, the more distinct levels of dialects are great.

Table 4. Confusion matrix without gender distinction with the combination of MFCC and $F 0$; a) $\mathrm{MFCC}=11$; b) $\mathrm{MFCC}=13$

\begin{tabular}{|c|c|c|c|c|}
\hline & ND & CD & SD & $\begin{array}{c}\text { Recognition } \\
\text { Rate }\end{array}$ \\
\hline ND & 824 & 220 & 206 & $65.9 \%$ \\
\hline CD & 178 & 932 & 140 & $74.6 \%$ \\
\hline SD & 140 & 258 & 852 & $68.2 \%$ \\
\hline
\end{tabular}

a)

\begin{tabular}{|c|c|c|c|c|}
\hline & ND & CD & SD & $\begin{array}{c}\text { Recognition } \\
\text { Rate }\end{array}$ \\
\hline ND & 826 & 226 & 198 & $66.1 \%$ \\
\hline CD & 152 & 965 & 133 & $77.2 \%$ \\
\hline SD & 158 & 229 & 863 & $69.0 \%$ \\
\hline
\end{tabular}

b)

\subsection{Combination of formants, corresponding bandwidths and $F 0$ parameters}

Normally, formant frequencies and bandwidths are vocal tract parameters. The formants are frequencies of vocal tract resonances. The first two formants are the most important because they decide the speech quality [14]. Formants and their bandwidths have been used for a lot of research on speech processing such as accent identification [15-17], speech recognition [18], speaker identification [19], study on genders and ethnical accents [20-22], dialect identification $[4,23-25]$.

In our experiments, the values of the first four formants and their bandwidths are calculated using Praat. These values are combined with $F 0$ and its variants. The experiments are performed using the baseline of Gaussian component number. The dialectal identification results with different combinations of these parameters are presented in Table 5 . The highest recognition rate is obtained for the case 7 . This recognition rate is higher than the one of the best case using MFCC $+F 0 s b M(t)$.

\footnotetext{
${ }^{1}$ www.praat.org
} 
Table 5. Recognition results using formants, corresponding bandwidths and $F 0$ parameters

\begin{tabular}{|c|c|c|}
\hline Index & $\begin{array}{l}\text { Formants+Bandwidths } \\
+F 0 \text { Parameters }\end{array}$ & $\begin{array}{l}\text { Recognition } \\
\text { Rate }\end{array}$ \\
\hline 1 & Formants+Bandwidths & $66.3 \%$ \\
\hline 2 & $F 0$ & $67.5 \%$ \\
\hline 3 & $\operatorname{diffF} 0(t)$ & $65.2 \%$ \\
\hline 4 & $c d F 0(t)$ & $67.0 \%$ \\
\hline 5 & $F 0 s b M M(t)$ & $67.8 \%$ \\
\hline 6 & $F 0 s b M(t)$ & $64.3 \%$ \\
\hline 7 & $F 0 \operatorname{sbMSD}(t)$ & $72.2 \%$ \\
\hline 8 & $\operatorname{LogF0}(t)$ & $71.6 \%$ \\
\hline 9 & $\operatorname{diff} \operatorname{LogF} 0(t)$ & $66.8 \%$ \\
\hline 10 & $\log F 0 \operatorname{sbMM}(t)$ & $67.7 \%$ \\
\hline 11 & $\operatorname{LogF} 0 \operatorname{sbM}(t)$ & $68.7 \%$ \\
\hline 12 & $\operatorname{LogF0sbMSD(t)}$ & $66.8 \%$ \\
\hline
\end{tabular}

\subsection{Effect of Gaussian component number on dialect recognition performance}

For this experiment, 13 MFCC coefficients $+F 0 s b M(t)$ are chosen and the Gaussian component number $M$ is taken from 20 (baseline) to 4096. GMM was trained and evaluated with this range of components. The DET (Detection Error Tradeoff) curves for different values of Gaussian component number are depicted in Figure 3.

From Figure 3, generally, the increase in $M$ increases the dialect recognition performance as we can also see in Table 6 .

Table 6. Average recognition rate with different values of Gaussian component number

\begin{tabular}{|c|c|}
\hline Gaussian component number & Recognition rate \\
\hline 20 & $70.8 \%$ \\
\hline 32 & $72.1 \%$ \\
\hline 64 & $71.5 \%$ \\
\hline 128 & $74.0 \%$ \\
\hline 256 & $73.2 \%$ \\
\hline 512 & $73.9 \%$ \\
\hline 1024 & $74.9 \%$ \\
\hline 2048 & $75.1 \%$ \\
\hline 4096 & $74.6 \%$ \\
\hline
\end{tabular}

The maximum recognition rate is $75.1 \%$ when $M$ equals 2048. In Figure 3, the points indicated by ${ }^{o}$ 's are weighted averages of the missed detection and false alarm rates or the minimum values of the Detection Cost Function $(D C F)$. These values are calculated as the following [26]:

$$
D C F=C_{m i s s} \cdot P_{m i s s} \cdot P_{t r u e}+C_{f a} \cdot P_{f a} \cdot P_{f a l s e},
$$


where $C_{m i s s}$ is the cost of a miss (rejection), $C_{f a}$ is the cost of an alarm (acceptance), $P_{t r u e}$ is the a priori probability of the target, $P_{f a}$ is the false alarm probability and $P_{\text {false }}=1-P_{\text {true }}$. $C_{m i s s}=C_{f a}=1$. The minimum value of the $D C F$ for $M=2048$ corresponds to the point which is closest to the origin.

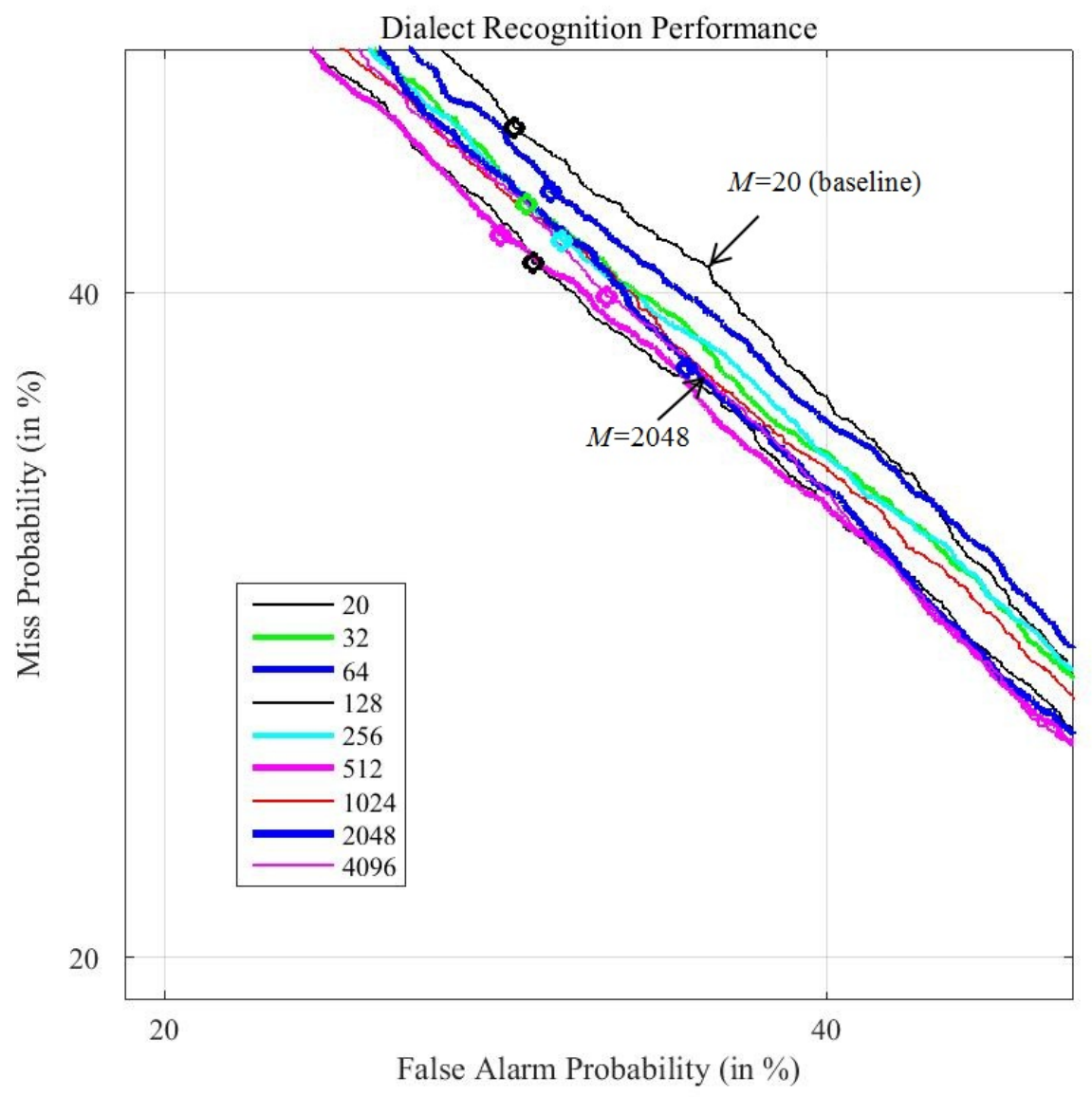

Figure 3. DET curves with Gaussian component number from 20 to 4096

\section{CONCLUSIONS}

Vietnamese is a tonal language and the dialects of Vietnamese are very rich in terms of phonetics and local vocabulary. In fact, based on the difference of pronunciation modality especially for F0 variation, one can discriminate three principal Vietnamese dialects such as northern, central and southern dialects. Therefore, by the combination of MFCC and F0 parameters for GMM model, the recognition rate of these Vietnamese dialects is improved significantly. The experiments show that to get the best score in order to receive the appropriate GMM model for dialect identification, the number of MFCC coefficients should be 13. Combining the first four formants, their bandwidths, and variants of the fundamental 
frequency, the recognition rate is generally higher than the case using MFCC and variants of the fundamental frequency. These research results can continue to develop for its application in the automatic recognition systems to enhance Vietnamese recognition performance.

\section{REFERENCES}

[1] B. Kortmann, A comparative grammar of British English dialects: agreement, gender, relative clauses. Walter de Gruyter, 2005, vol. 1.

[2] J. Li, T. F. Zheng, W. Byrne, and D. Jurafsky, "A dialectal chinese speech recognition framework," Journal of Computer Science and Technology, vol. 21, no. 1, pp. 106-115, 2006.

[3] S. Aunkaew, M. Karnjanadecha, and C. Wutiwiwatchai, "Development of a corpus for southern thai dialect speech recognition: Design and text preparation," in Symposium on Natural Language Processing (SNLP 2013), Phuket Thailand, 2013.

[4] S. Sinha, A. Jain, and S. S. Agrawal, "Acoustic-phonetic feature based dialect identification in hindi speech." International Journal on Smart Sensing Ë Intelligent Systems, vol. 8, no. 1, 2015.

[5] H. T. Chau, "Vietnamese dialects," 2009.

[6] J.-F. Bonastre, F. Wils, and S. Meignier, "Alize, a free toolkit for speaker recognition." in ICASSP (1), 2005, pp. 737-740.

[7] P. A. Torres-Carrasquillo, T. P. Gleason, and D. A. Reynolds, "Dialect identification using gaussian mixture models," in ODYSSEY04-The Speaker and Language Recognition Workshop, 2004, pp. 297-300.

[8] B. Ma, D. Zhu, and R. Tong, "Chinese dialect identification using tone features based on pitch flux," in 2006 IEEE International Conference on Acoustics Speech and Signal Processing Proceedings, vol. 1. IEEE, 2006, pp. I-I.

[9] W. M. Campbell, E. Singer, P. A. Torres-Carrasquillo, and D. A. Reynolds, "Language recognition with support vector machines," in ODYSSEY04-The Speaker and Language Recognition Workshop, 2004, pp. 41-44.

[10] P. A. Torres-Carrasquillo, E. Singer, M. A. Kohler, R. J. Greene, D. A. Reynolds, and J. R. Deller Jr, "Approaches to language identification using gaussian mixture models and shifted delta cepstral features." in INTERSPEECH, 2002, pp. 32-36, 82-92.

[11] F. Biadsy, J. Hirschberg, and D. P. Ellis, "Dialect and accent recognition using phoneticsegmentation supervectors." in INTERSPEECH, 2011, pp. 745-748.

[12] P. N. Hung, T. V. Loan, N. H. Quang, and P. Q. Hung, "Identification of vietnamese dialects using gmm," in Proceedings of the 6th National Conference on Fundamental and Applied Information Technology Research (FAIR6), vol. 1. ISBN 978-604-913-165-3, 2014, pp. 449-452.

[13] P. N. Hung, T. V. Loan, and N. H. Quang, "Corpus and statistical analysis of f0 variation for vietnamese dialect identification," in The 3rd International Conference on Computer and Computing Science Proceedings, Hanoi, Vietnam (COMCOMS 2015), vol. 111. ISSN: 22871233 ASTL, 2015, pp. 205-210.

[14] R. Carlson, G. Fant, and B. Granström, "Two-formant models, pitch, and vowel perception," Acta Acustica united with Acustica, vol. 31, no. 6, pp. 360-362, 1974. 
[15] D. Stantic and J. Jo, "Accent identification by clustering and scoring formants," World Academy of Science, Engineering and Technology, vol. 6, no. 3, pp. 379-384, 2012.

[16] A. Hanani, M. J. Russell, and M. J. Carey, "Human and computer recognition of regional accents and ethnic groups from british english speech," Computer Speech $\&$ Language, vol. 27, no. 1, pp. $59-74,2013$.

[17] K. Mannepalli, P. N. Sastry, and V. Rajesh, "Accent detection of telugu speech using prosodic and formant features," in Signal Processing And Communication Engineering Systems (SPACES), 2015 International Conference on. IEEE, 2015, pp. 318-322.

[18] P. N. Garner and W. J. Holmes, "On the robust incorporation of formant features into hidden markov models for automatic speech recognition," in Acoustics, Speech and Signal Processing, 1998. Proceedings of the 1998 IEEE International Conference on, vol. 1. IEEE, 1998, pp. 1-4.

[19] T. Becker, M. Jessen, and C. Grigoras, "Forensic speaker verification using formant features and gaussian mixture models." in Interspeech, 2008, pp. 1505-1508.

[20] M. Yusnita, M. Paulraj, S. Yaacob, M. N. Fadzilah, and A. Shahriman, "Acoustic analysis of formants across genders and ethnical accents in malaysian english using anova," Procedia Engineering, vol. 64, pp. 385-394, 2013.

[21] M. P. Gelfer and V. A. Mikos, "The relative contributions of speaking fundamental frequency and formant frequencies to gender identification based on isolated vowels," Journal of Voice, vol. 19, no. 4, pp. 544-554, 2005.

[22] J. M. Hillenbrand and M. J. Clark, "The role of $\mathrm{f} 0$ and formant frequencies in distinguishing the voices of men and women," Attention, Perception, \&5 Psychophysics, vol. 71, no. 5, pp. 1150-1166, 2009.

[23] R. Hagiwara, "Dialect variation and formant frequency: The american english vowels revisited," The Journal of the Acoustical Society of America, vol. 102, no. 1, pp. 655-658, 1997.

[24] E. Jacewicz and R. A. Fox, "The effects of dialect variation on speech intelligibility in a multitalker background," Applied Psycholinguistics, vol. 36, no. 03, pp. 729-746, 2015.

[25] R. A. Fox and E. Jacewicz, "Cross-dialectal variation in formant dynamics of american english vowels," The Journal of the Acoustical Society of America, vol. 126, no. 5, pp. 2603-2618, 2009.

[26] A. Martin, G. Doddington, T. Kamm, M. Ordowski, and M. Przybocki, "The det curve in assessment of detection task performance," DTIC Document, Tech. Rep., 1997.

Received on March 17 - 2016

Revised on July 13 - 2016 